\title{
Glyco-biomarkers: Potential determinants of cellular physiology and pathology ${ }^{1}$
}

\author{
Azita Alavi* and John S Axford \\ The Sir Joseph Hotung Centre for Musculoskeletal Diseases, St George University of London, London, UK
}

\begin{abstract}
Once dismissed as just the icing on the cake, sugar molecules are emerging as vital components in life's intricate machinery. Our understanding of their function within the context of the proteins and lipids to which they are attached has matured rapidly, and with it the far reaching clinical implications are becoming understood.

Recent advances in high-throughput glycomic techniques, glyco biomarker profiling, glyco-bioinformatics and development of increasingly sophisticated glyco-arrays, combined with our increased understanding of the molecular details of glycosylation have facilitated the linkage between aberrant glycosylation and human diseases, and highlighted the possibility of using glycobiomarkers as potential determinants of disease and its progression.

The focus of this review is to give an insight into the biological significance of these glycomodifications, highlight some specific examples of glyco-biomarkers in relation to autoimmunity and in particular rheumatoid arthritis, and to explore the exciting possibility of exploiting these for diagnostic and prognostic strategies.
\end{abstract}

Keywords: Glycosylation, glycomics, glyco biomarkers, autoimmunity, sugar printing, glyco-antigens, immunoglobulin G, rheumatoid arthritis

\section{Introduction}

The recent surge of interest in glycomics and the search for glyco-biomarkers of disease stems from the fact that glycosylation has, finally, gained recognition for the pivotal role that it plays in virtually all aspects of our system; from embryogenesis to pathogenesis [1-6]. This comes as no surprise, since the surface of our entire cellular network, as well as those of pathogens, and the backbone of most proteins and lipids, is decorated with a dense complement of either linear or intricately branched complex sugar structures [3,5,7].

These glycans constitute the most abundant and diverse of the post-translation modifications in our sys-

\footnotetext{
${ }^{1}$ Disclosure statement: The authors have been recipients of research grants from the Arthritis Research Campaign, European Commission, Abbott Pharmaceuticals, Lupus UK, Mannatech Inc and various Charitable Donations.

*Corresponding author: A. Alavi, The Sir Joseph Hotung Centre for Musculoskeletal Diseases, St George University of London, London, SW17 ORE, UK. Tel.: +44 208266 6802; E-mail: aalavi@sgul.ac.uk.
}

tem and are therefore an integral feature of almost all biomolecules including nearly all cell surface and over $70 \%$ of secretory proteins, as well as glycolipids, glycosphingolipids (such as the ABO histo-blood group antigens), lipopolysaccharides, and glycosaminoglycans (GAGs; such as heparin). In the majority of these examples the glycan moiety constitutes a substantial portion of the mass, size and charge of the glycoconjugate, and can thus exert considerable inter- and intramolecular effects. As such glycans have the potential to generate extensive physical and biochemical diversity (through the formation of large numbers of glycoforms), and therefore confer considerable coding capacity for relay of biospecific information. And so, in addition to performing a structural and protective role, a large number of glycans have important functional roles as specific information tags or recognition epitopes $[3,8]$.

The information coded into these, spatially accessible, sugar epitopes is decoded by a sophisticated recognition system, which is comprised of a large cohort of carbohydrate binding proteins that include lectins, 
Table 1

A list of some of the endogenous lectins/carbohydrate-recognizing proteins expressed in our system. Depending on their structure and mode of action, lectins are subdivided in several groups. These lectins contain one or more carbohydrate recognition domains that determine their specificity.

\begin{tabular}{|c|c|}
\hline Endogenous lectins & Function \\
\hline Annexins & $\begin{array}{l}\text { Functions include binding to carbohydrate moieties of sialoglycoproteins and GAGs. Examples include annexin } \\
\text { IV, in kidney and pancreas. May be important apical sorting (secretory vesicles) and exocrine-type neurotrophic } \\
\text { activity and in cell-adhesion (or inhibition of cell-adhesion). }\end{array}$ \\
\hline $\begin{array}{l}\text { C-type lectins: calcium- } \\
\text { dependent Soluble/ } \\
\text { transmembrane }\end{array}$ & $\begin{array}{l}\text { Collectins; soluble lectins such as C- reactive protein (CRP), mannose binding lectin (MBL), surfactants SP-A } \\
\text { and SP-D and ficolins, which have the capacity to activate complement and thus play an important role in innate } \\
\text { immunity as well as autoimmunity } \\
\text { Selectins; membrane bound with specific function in leukocyte adhesion to endothelial cells through sialyl- } \\
\text { LewisX recognition; therefore important in both normal physiology and in inflammation and immunity to tumor } \\
\text { and virally infected cells. } \\
\text { The selectin on the leukocyte side is L-selectin while those on the endothelial side are E- and P-selectins } \\
\text { Type I receptors; includes cell surface mannose receptors on macrophages and other types of cells, and DEC-205 } \\
\text { on dendritic cells. Involved in molecular uptake into cells. } \\
\text { Type II receptors; typical examples are sialoglycoprotein receptor of hepatocytes, macrophage galactose/N- } \\
\text { acetylgalactosamine specific lectin, natural killer cell receptors and low affinity IgE receptor (CD23). These are } \\
\text { either involved in the molecular uptake into cells through the endocytic pathway or in the signal transduction } \\
\text { based on cell-cell recognition. }\end{array}$ \\
\hline I- type lectins & $\begin{array}{l}\text { I (immunoglobulin)-type lectins, have } 2 \text { domains; carbohydrate-binding and an Ig-like domain. Examples } \\
\text { include siglecs, which recognize sialic acid, and are expressed on specific subsets of tissue-phase or activated } \\
\text { macrophages }\end{array}$ \\
\hline P-type lectins & Involved in trafficking of lysosomal enzymes \\
\hline $\begin{array}{l}\text { S-type lectins; also } \\
\text { known as Galectins }\end{array}$ & $\begin{array}{l}\text { A rapidly growing family of metal-independent lectins with diverse histological localization, in cytoplasm, } \\
\text { nuclei, cell surfaces and extracellular spaces; depending on the galectin species. } \\
\text { They share galactose-specificity and display potent biological activities, such as the ability to induce apoptosis, } \\
\text { or metabolic changes, such as cellular activation and mitosis. Examples include galectin } 1 \text { which induces T cell } \\
\text { apoptosis and galectin } 3 \text {, associated with tumours, which inhibits apoptosis }\end{array}$ \\
\hline
\end{tabular}

collectins, adhesion molecules, and anti-carbohydrate antibodies (Table 1) [8-12]. This versatile carbohydrate recognition system combined with our extensive glycome are key players in orchestrating the complex functional network of bimolecular interactions that coordinate molecular and cellular function in relation to innate and adaptive immunity [13-19].

Given the diversity of structures and functions, and the potential for conveying information essential to maintenance of immune homeostasis, it is not surprising that the role of glycosylation in the development, regulation, and progression of disease has come under increased scrutiny [6].

\section{Physiological diversity and function of glycans}

As might be imagined from their ubiquitous nature, and their ability to convey information, the biological roles of glycans are formidable and span the complete spectrum, from those that are relatively subtle (e.g. structural) to those that are critical (e.g. crucial for the development, function and survival of an organism).

However, the elucidation of a specific physiological role for a given glycan modification(s) poses a formidable challenge. This is because glycan structures can play different roles in different cells/tissues, at different times [3,20-22], and also because in some instances what is deemed structural under normal physiological conditions may be rendered antigenic in certain disease conditions. Examples of these include 1) the developmentally regulated expression and distribution of the $\mathrm{ABO}$ histo-blood group antigens [23], and 2) the glycosylation changes that render certain collagen epitopes arthrogenic [24].

The plethora of biological functions ascribed to glycans include mechanisms of protein folding and turnover [25], trafficking and distribution [26,27], pharmacokinetics [28], as well as immunogenicity; where glycomodifications may lead to unmasking of antigenic epitopes in the glyconcjugate backbone (e.g. antigenic peptide sequences) or reveal/create glyco-antigenic determinants (through aberrant exposure of certain terminal sugar residues e.g. $N$-acetylglucosamine, or via aberrant changes to the core structure e.g. unusual chain elongation/branching) $[29,30]$.

Other functions ascribed to glycans include their role as ligands for specific receptors in areas such as signaling [31], immuno-modulation [32,33], cell communication and adhesion [34-36], including those involved 
in tumor progression and metastasis [11], and as points of attachment for pathogens [8,37-40].

The latter may be of particular relevance as it adds credence to the emerging, sometimes controversial, linkage between blood group antigen expression and disease [41-46], in which certain ABO antigens are implicated because of their role as microbial glycan receptors, as tumor antigens and as ligands for important immunologic reactions $[5,47,48]$.

Glycans are also important components of cytosolic and nuclear proteins. An important example of this is the dynamic modification of these proteins with $O$ linked $\beta$ - $N$-acetylglucosamine $(O$-GlcNAc). The covalent attachment of this $O$-GlcNAc (to serine or threonine residues) has been shown to be a regulatory posttranslational modification that is responsive to various stimuli. Its main function is that of a regulatory switch in the metabolic control of signal transduction, transcription, stress response, apoptosis, as well as T- and B-lymphocytes activation [17,49]. O-GlcNAc glycosylation is also critical in both neuronal function and dysfunction (neuronal signaling and synaptic plasticity) and may have a crucial impact on the nervous system and consequently various neurodegenerative diseases [50].

An appreciation of the importance of glycans and their multidimensional roles in various physiological and pathological circumstances would be incomplete without some degree of insight into their biosynthesis and the factors that determine their structural complexity and diversity, as discussed below.

\section{Determinants of cellular physiology/pathology}

A unique feature of glycosylation is the fact that despite its complexity and precise nature the biosynthesis of glycans can not be directly predicted from the DNA template [Fig. 1], but is instead governed by an elaborate mechanism that utilizes a multitude of glycoenzymes [21]. These enzymes, which display exquisite biological specificity, are expressed in a cell/tissuespecific, and temporally regulated manner. Their expression is controlled by multiple tissue-specific promoters that may be activated/suppressed under different physiological circumstances (e.g. expressed at discrete points in lymphocyte development and peripheral activation) [35]. Examples of this include the way in which pro-inflammatory or anti-inflammatory cytokines alter the expression of specific glycosyltransferases; that in turn regulate the conversion of activated
$\mathrm{T}$ cells into memory cells, or the differentiation of $\mathrm{Th}$ cells into Th1 and Th2 subsets and thereby influence disease outcome [18,51].

It is therefore not surprising that the glyco-profile of a given cell/tissue (including serum) can alter in response to a whole host of physiological $[40,52,53]$ or pathological situations e.g. angiogenesis, immune challenge, inflammation or oncogenic transformation and metastasis $[3,8,11,54]$.

An understanding of this code as it relates to disease states, at both molecular and functional levels can help unravel disease mechanisms and thus pathology.

\section{Glycosylation and disease}

In accord with the above observations, aberrant changes in cellular processes, such as those that accompany disease, are therefore likely to result in alterations of the glycan profiles of the cell surface and/or secreted glycoconjugates, in particular glycoproteins. And so, not surprisingly, most major diseases, when probed, are found to be directly/indirectly associated with a change in the glycosylation pattern of at least one central structure.

This has led to the novel concept of glyco-biomarkers and "Sugar profiling", which was first introduced by our group in relation to the study of IgG glycosylation changes in rheumatoid arthritis (RA) [55-58] and other rheumatological diseases such as systemic lupus erythematosus (SLE), but can now be extended to study diseases as diverse as asthma, acute respiratory distress syndrome, cystic fibrosis [40,59], neuropathology (including Creutzfeldt-Jakob disease) [60,61], muscular dystrophy [62], cardiovascular disorders such as atherosclerosis [63], endocrinology and diabetes [64], inflammatory bowel disease [65], IgA nephropathy (IgAN) [66,67], nephrolithiasis [68], and last but not least, almost all forms of malignancy [69-71].

The glycosylation changes in relation to these and many other diseases, not covered here, range from the subtle to the palpable and can be acquired/inherited.

\subsection{Inherited glycosylation diseases; rare or under diagnosed?}

Given the critical role of glycosylation, all the inherited glycosylation diseases detected so far are autosomal recessive disorders that are due to polymorphisms and minor mutations and never the result of gross mutations (which are unlikely to occur as they would be 


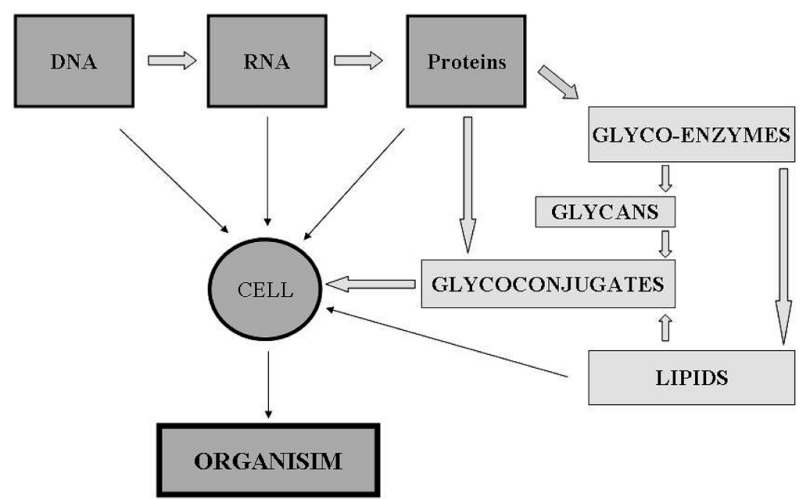

Fig. 1. The biosynthesis of glycans can not be directly predicted from the DNA template, but is instead governed by a complex glyco-enzyme directed mechanism.

fatal at the early stages of fertilization/embryogenesis). Those that occur are relatively rare, and give rise to either severe phenotypic consequences (sometimes with neonatal death) or very minor ones; which are likely to be asymptomatic. The underdiagnosis of this group of diseases is further compounded by ascertainment bias, due to pleiotropic or unpredictable phenotypes.

The plethora of different mutations detected results in a diverse group of diseases including leukocyte adhesion deficiency syndrome II, congenital dyserythropoietic anemia type II (also known as HEMPAS), and an emerging varied group of disorders $(>20$ separate genes and more than 100 allelic variants since the first report in 1980, the majority of which were identified in the past few years) collectively known as congenital disorders of glycosylation (CDGs) [72].

By far the most frequent of these is CDGIa. This group of diseases ( $>60$ mutations in phosphomannomutase-2) have a variable clinical spectrum, ranging from disorders restricted to specific organs to severe multisystemic disorders, including central nervous system phenotypes [72], suggesting that the brain is particularly susceptible to perturbations in glycosylation.

As these glycosylation disorders are likely to target and affect a large set of proteins/lipids, it is not hard to see how glycosylation enzymes might act as modifiers of other gene defects and thus modulate the severity and nature of diseases such as various neuropsychiatric conditions including Alzheimers and Schizophrenia [61].

The diseases discovered so far, however, may represent only the "tip of the iceberg" [72,73], as it is estimated that defects in any of the well over 50-100, critical glycosylation genes will cause diseases such as those described above, and many of these can only be detectable by sugar profiling [74].

\subsection{Acquired glycosylation diseases}

Aberrant, non-inherited, glycomodifications are extensive and invariably found to be associated with, or a pre-requisite for, a wide-ranging myriad of disease phenotype [2-4,6].

The most obvious of these are changes that accompany angiogenesis or disease associated immune processes such as cellular activation, recruitment and inflammation; all of which encompass sugar changes of one type/another e.g. glycomodification of various activation/co-stimultory cell surface molecules on cytotoxic/helper $\mathrm{T}$ cells, as well as various ligands such as sLex [40], which act as tissue specific zip codes regulating lymphocyte traffic to a given site/organ $[3,75$, 76].

However, in addition to these, there is also a catalogue of "disease specific glycosylation changes" that play a key role in the actual disease mechanism, and are thus of pathophysiological significance $[3,33,58$, $77,78]$.

These fall into two categories: In some instances, as for example cancer, altered glycosylation is a universal feature that reflects significant changes in certain specific glyco-enzymatic pathways and thus closely correlates with critical aspects of the disease, whilst in others the glycomodifications may be more subtle, acting as a trigger that could instigate biological effects that may initiate or, in certain circumstances, alter the course of disease.

The impact of these glycosylation changes in relation to autoimmunity and in particular RA pathology will be the main focus of this section, followed by a brief overview of a few select examples of other autoimmune diseases/disease mechanisms where specific glycosylation changes play a critical role. 


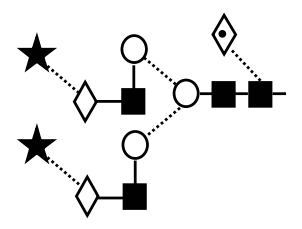

Sialylated, outer arm fucosylated, biantennary $N$-glycan: alpha-Neu5Ac-( $(2 \rightarrow 6)$-beta-Gal-( $1 \rightarrow 4)$-beta-GlcNAc- $(1 \rightarrow 2)$-alpha-Man-( $(1 \rightarrow 6)$-[alpha-Neu5Ac- $(2 \rightarrow 6)$-betaGal-( $(1 \rightarrow 4)$-beta-GlcNAc- $(1 \rightarrow 2)$-alpha-Man- $(1 \rightarrow 3)$ ]-beta-Man- $(1 \rightarrow 4)$-beta-GlcNAc- $(1 \rightarrow 4)$-[alpha-Fuc-( $(1 \rightarrow 6)]$-beta-GlcNAc.
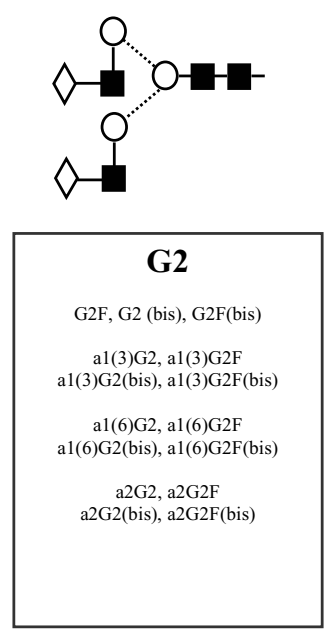
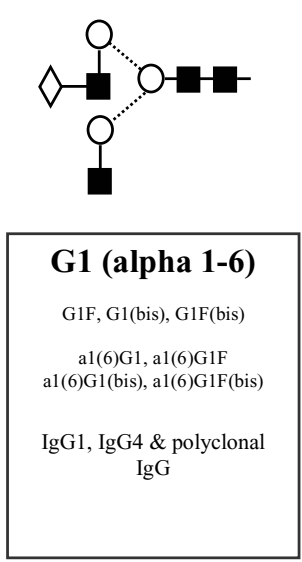
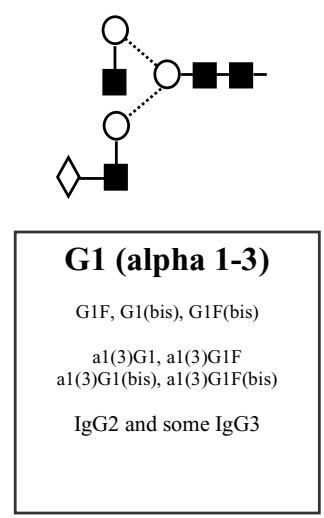
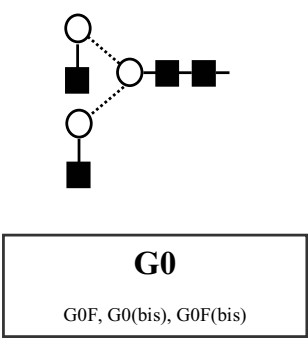

Fig. 2. The 36 complex biantennary N-glycan variants associated with the single glycosylation site on the Fc moiety of IgG. Glycans are designated as G2, G1 and G0 (according to the number of terminal galactose residues), followed by bis (bisecting $N$-acetylglucosamine and/F (Fucose), and a1 and or a2 (according to the number of terminal sialic acid residues). Monosialylation and monogalactosylation may occur in either $\alpha$-6/alpha 1-3 configurations. Key for glycan structures: $\mathbf{\square} ; N$-acetylglucosamine (GlcNAc), $\bigcirc$; mannose (Man), $\diamond$; galactose (Gal), $\star$; N-acetylneuraminic acid (NeuNAc), $\odot$; fucose (Fuc), —; beta linkage, and; - - -; alpha-linkage.

\section{Glyco-biomarkers and autoimmunity}

Glycomodification may represent one way in which immune tolerance can be bypassed. Some posttranslational modifications can create new self antigens (Ags) or even mask Ags normally recognized by the immune system [24,30,79-82]. The former is of particular relevance since even subtle changes may lead to immune activation resulting from recognition of the glyco-Ags by the naturally occurring anti-glycan auto antibodies (AutoAbs); directed against a vast repertoire of non-self glycan structures [83] existing on bacterial, fungal and parasite cells.

As such the link between glycomodifications and autoimmunity is complex and includes:

(i) Cross-reactivity: Unlike classical peptide epitopes, glyco epitopes (glycotopes) can share significant structural homologies. As such they can display extensive cross-reactivity and thus behave as "panepitopes", which may, in some contexts, be implicated in autoimmunity. A classic example of this is the possible recognition of abnormally exposed $N$ Acetylglucosamine (GlcNAc) in our system (resulting from aberrant hypogalactosylation; as seen in RA IgG) by pathogen associated molecular pattern receptors of innate immunity such as mannose binding lectin (MBL), which may result in immune dysregulation [9].

(ii) Neo-expression: Expression of glycans normally restricted to other tissues or molecules [84] or incorporation of immunogenic non-human dietary glycans e.g. $N$-Glycolyneuraminic acid [85], which could generate xenoreactive, and potentially autoreactive $\mathrm{Ab}$ responses and cause long-term inflammatory reactions. 
(iii) Inappropriate processing and presentation: In some instances glycan changes can lead to altered processing and presentation of selfantigens to $\mathrm{T}$ cells via major histocompatibility complex (MHC). An interesting example of this is the role of collagen derived remnant glycotopes in autoimmune arthritis [24].

(iv) Conformational changes/unmasking of possible antigenic epitopes: Glycomodifications can result in the exposure of possible antigenic peptide sequences that would have otherwise been masked by the sugar moiety, or give rise to localized conformational changes in both the glycan moiety and the polypeptide, which could render the molecule antigenic; as in immunoglobulin A ( $\operatorname{IgA}$ )/immunoglobulin $\mathrm{G}$ $(\mathrm{IgG})$ in $\operatorname{Ig} \mathrm{A}$ nephropathy and RA respectively $[2,3,58,66,86]$.

The latter has been the focus of extensive studies as will be discussed below.

\subsection{IgG glyco-biomarkers and rheumatoid arthritis}

The $N$-linked glycans at the single, highly conserved, glycosylation site in the constant domain of IgG Fc region (which can be present in any one of 30 variations depending on the presence/absence of absence of galactose, sialic acid, bisecting $N$-acetylglucosamine [bis-GlcNAc] and fucose; Fig. 2) are critical features, which have far reaching structural and functional impact. Affecting both the innate and adaptive arms of the immune response $[33,87,88]$.

During the past two decades particular emphasis has been placed on the aberrant glycosylation of $\mathrm{IgG}$ and its role in RA pathogenesis [3,57,58,89-94]. The research in this field has been extensive and has drawn together all aspects of aberrant IgG glycosylation, including the structural anatomy of the IgG, the clinical implications in both human disease and animal models, the glycosylation enzymes and the genes that encode and regulate these enzymes, and the possible pathogenic mechanisms of glyco-modified IgG [3,21,24,57,58,86].

The enormous volume of data generated suggests that RA may be a dysregulated glycosylation disease in which IgG glycomodifications may play a pivotal role. These glycomodifications have been shown to be far more complex than previously thought, encompassing not only galactosylation, but also fucosylation and sialylation, as well as the additional microheterogeneity of both symmetrical and asymmetrical pairing of the Fc glycans $[3,58,95]$.
The loss of galactose and the increased levels of core fucosylation affect the interface between IgG-Fc fragments and its interaction with other molecules and consequently interfere with $\mathrm{Fc}$ receptor binding and effector activities [88] causing defective/altered effector/regulatory mechanisms of the immune and thus inflammatory response [3,24,58].

\subsubsection{IgG Glyco-biomarkers as useful diagnostic tools for $R A$}

The rheumatic diseases are heterogeneous (Fig. 3), and overlapping disease syndromes may be found [57]. At present there is no single diagnostic test capable of differentiating one disease from another. Increased levels of IgG-G0 have proven to be useful biomarkers of RA. Their presence in serum predates the onset of RA by at least 10 years, and in early synovitis has been found to be associated with the development of RA; such that when combined with RF it has $90 \%$ sensitivity, $95 \%$ specificity and $94 \%$ positive predictive value [3]. This has prompted the use of sugar printing for the differentiation of rheumatic diseases [55-57]. Sugar printing of serum IgG can differentiate early rheumatoid arthritis (ERA) and RA from each other and from other rheumatic diseases and hence may constitute a relatively rapid diagnostic test for patients presenting with arthritis [57]. In the case of RA, it was found that ERA/RA are distinguished from other diseases by their IgG-G0 and -G0F (the most abundant G0 structure in RA), but differ from each other by their monogalactosyl (G1) and sialylated sugar profiles. Undifferentiated arthritis, in contrast, had no specific distinguishing features, as one would expect from what is probably a heterogeneous group of pathologies. The strength of the association with RA is confirmed by the fact that G0 and G0F can be used to predict RA from the pool of patients with a broad spectrum of disease. Although the study was not designed for the purpose of evaluating the diagnostic utility of IgG-G0/-G0F per-se, over $75 \%$ of RA patients were correctly identified as having the disease and the test was shown to have a sensitivity of $50 \%$, and a specificity of $84 \%$ [57].

Structural studies on IgG oligosaccharides have also proved useful in unraveling some of the clinical overlap in patients with primary Sjogren's syndrome (SS); whereby the appearance of IgG-G0 in primary SS may be related to future complication with RA [96].

Clinical studies in this field strongly support the proposed relationship between IgG glycosylation, immune complex formation, increased rheumatoid factor (RF) avidity, as well as MBL binding and pathology in RA. 


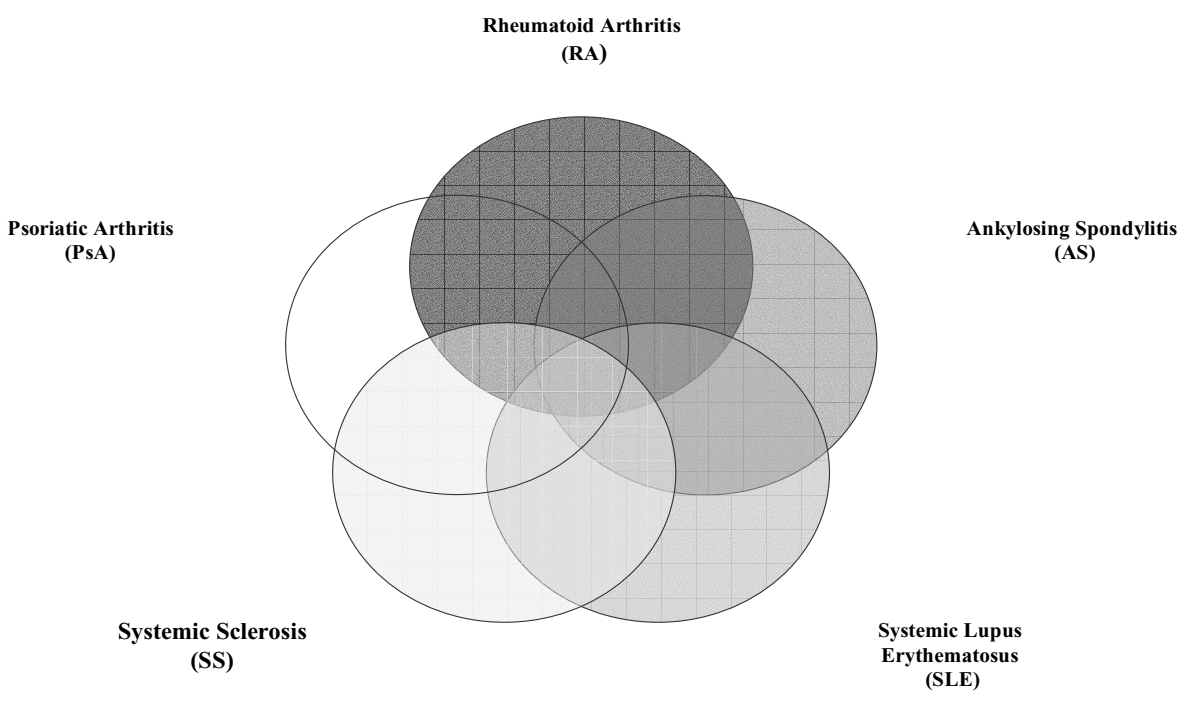

Fig. 3. The rheumatic diseases are heterogeneous, and overlapping disease syndromes may be found. Many patients diagnosed with autoimmune rheumatic disease cannot be categorized easily into one of the established clinical entities such as SLE or systemic sclerosis. IgG glyco-biomarkers may be useful in terms of clarifying diagnosis and prognosis, and facilitating disease management.

They demonstrate that increased IgG-G0 (circulating in the serum and/or in immune complexes in the synovial fluid), correlate with increased RF avidity, with higher tender joint score, and a higher frequency and number of subcutaneous nodules in RA patients [90,97-99].

\subsubsection{IgG Glyco-biomarkers as useful prognostic tools for $R A$}

In addition, IgG-G0 has also proved to be a good prognostic indicator of RA. High incidence of serum IgG-G0 is related to disease activity and the progression to erosive articular changes, as determined in a well-characterized cohort of 127 female RA patients who were followed for a mean duration of 6 years. IgG-G0 values correlated with the number of erosions, disease activity and served as an indicator for disease progression [3,57,58,98].

IgG-G0 not only correlates with severity and duration of disease [90], but has also been shown to return to normal levels following treatment e.g. with anti-TNF [100]. The latter observation is in-line with changes observed in pregnancy where the decrease in IgG-G0 levels is associated with a remission in the disease, and where a rapid rebound increase in IgG-G0, post partum, is associated with disease flares in RA patients [91]. The latter is of particular importance as it further supports the notion that IgG-G0 may be a susceptibility factor in the development of RA.

In this respect IgG glyco-biomarkers may be very useful in determining the clinical efficacy of im- munotherapeutic agents such as the new biologics [100], which are revolutionizing the way that we treat autoimmune diseases such as RA and SLE [3].

\subsection{IgG glyco-biomarkers in other rheumatological diseases}

The appearance of IgG-G0 is also a general feature of other unrelated chronic granulomatous diseases e.g. Crohn's disease (CD) and Mycobacterium tuberculosis, as well as a restricted group of other rheumatological diseases such as SLE, SS, psoriatic arthritis and juvenile idiopathic arthritis (JIA) [56]. However, detailed sugar profiling of these diseases has demonstrated differential patterns of Fc-glycomodifications; encompassing Gal, Fuc, Bis-GlcNAc and sialic acid; and supports the notion that each disease may be associated with a distinct pattern of IgG glycosylation [3,55-58].

Glycosylation-related pathology is not unique to $\mathrm{IgG}$, and can include other immunoglobulins (e.g. IgA) and other immunologically pertinent molecules such as the acute phase proteins, as well as mucins, as summarized below.

\subsection{IgA glyco-biomarkers in IgAN and Henoch-Schönlein Purpura (HSP)}

$\operatorname{Ig} \mathrm{AN}$ is defined by the deposition of $\operatorname{Ig} \mathrm{A} 1$ in the glomerular mesangium, whilst HSP is a form of systemic vasculitis characterized by tissue deposition of 
IgA. Nephritis with IgA1 deposition is a common feature of HSP and histologically indistinguishable from IgAN. Analysis of serum IgA1 from patients with the IgA nephropathy showed decreased galactosylation (and hence sialylation) of the O-glycans in the hinge region of $\operatorname{IgA} 1$ ( $\operatorname{Ig} \mathrm{A} 1$ has 5 sets of closely located Oglycans in this region). This hypogalactosylation corresponds to decreased B cell $\beta 13$-galactosyltransferase activity, which may be altered due to increased production of Th2 cytokines [66,101].

Altered glycosylation of $\operatorname{IgA} 1$ results in a loss of conformational rigidity, which may explain the increased formation of aggregates, and the glomerular accumulation of $\operatorname{IgA} 1$ [66].

\subsection{Glyco-biomarkers in Inflammatory Bowel Disease (IBD)}

IBD is a chronic intestinal disorder comprising two major types: ulcerative colitis (UC) and CD; with considerable overlap. The diagnosis and the differentiation between UC and CD is therefore invariably dependent on a combination of clinical, serological, endoscopic, histopathologic and radiological characteristics; with $>15 \%$ of patients being diagnosed with indeterminate colitis. The degree of glycosylation of the mucins is central to their role in IBD. It has been demonstrated that the degree of sulphation and sialylation and the length of the oligosaccharide chains can vary, and thus affect the function of mucins as a protective barrier. The changes are different in UC compared to CD. In UC, the sialic acids of the colonic mucosa, which are normally heavily O-acetylated, lose this modification [102]. This may have pathogenic significance as these modifications do render the sialic acids more resistant to bacterial sialidases.

\subsection{Anti-glycan Auto-antibodies (AutoAbs) as biomarkers of disease}

As already pointed out glycan structures share significant structural homologies and can act as cross reactive panepitopes. These may in certain circumstances be highly antigenic and instigate the activation and increased production of naturally occurring autoAbs, which may result in inflammation and autoimmunity.

\subsubsection{Auto-abs against IgG-GO as biomarkers in RA and JIA}

The increased IgG-G0 in RA has been shown to be associated with presence of increased levels of anti IgGG0 auto-Abs. Studies examining the diagnostic value of anti IgG-G0 auto-Abs, in 266 Japanese patients with systemic autoimmune diseases, including 60 with RA, suggests that anti IgG-G0 Abs may be a more specific marker for RA than conventional IgM RF [97], which is the current biomarker of choice for the diagnosis of RA.

These anti IgG-G0 autoAbs, show a significant correlation with $\mathrm{C}$ reactive protein levels and have a higher sensitivity in detecting immunological disorders in JIA and juvenile onset Sjogren's syndrome when compared with RF [97,103].

\subsubsection{Auto-abs against neuronal glycans in certain neuropathies}

A number of neuropathies are associated with circulating auto-Abs directed against certain glycan epitopes that are highly expressed in the nervous system (e.g. the sugar chains of gangliosides), resulting in autoimmune nerve damage [78]. Anti-ganglioside IgM Abs can cause leakage of the blood-nerve barrier in a concentration-dependent and complementindependent manner, and can also bind to neuronal gangliosides (to create a neuromuscular block) and serve as a marker of axonal damage in neuropathies such as multiple sclerosis [104].

The majority of these auto-Abs originate either from our naturally occurring pool of anti-glycan Abs (B cell clones; usually germ-line encoded), or are the result of exposure to bacterial antigens. Examples include:

i) Monoclonal IgM or IgA Abs; highly specific for either ganglio-series gangliosides, or sulfated glucuronosyl glycans (the so-called HNK-1 epitope), secreted by benign or malignant B-cell neoplasms [105]. The presence of these Abs is associated with the onset of symptoms of a demyelinating neuropathy involving the peripheral and central nervous systems: the GuillainBarre and Miller-Fisher syndromes, respectively.

ii) Cross reactive AutoAbs; directed against gangliosides structures such as $\mathrm{G}_{M 1}$ and $\mathrm{G}_{Q 1 b}$, which occur following infection with bacteria such as Campylobacter jejuni, which mimic ganglioside structures [106]. 


\subsubsection{Auto-Abs against Tn antigen in Tn-Polyagglutinin Syndrome}

Anti-Tn Abs are yet another example of naturally occurring anti-glycan Abs present in our sera. Tn polyagglutinability syndrome is an acquired condition where the blood cells made by the bone marrow express the Tn antigen; O-linked N-acetylgalactosamine (GalNAc $\alpha 1$ $\mathrm{O}-\mathrm{Ser} / \mathrm{Thr}$ ) and the sialylated-Tn (SA $\alpha 2,6$ GalNAc $\alpha 1$ O-Ser/Thr), thus becoming susceptible to hemagglutination by the naturally occurring anti-Tn Abs [107]. This change appears to be due to acquired stem-cellbased loss of expression of the O-glycan Core 1 $\beta 1-3$ galactosyltransferase activity, which occurs despite the fact that there may be as many as at least five distinct gene loci encoding additional copies of this enzyme. Patients with this syndrome show a wide range of symptoms. Some have varying degrees of hemolytic anemia and/or decreases in other blood cell types, whilst others have no detectable symptoms and are only picked up through blood typing. Although the mechanisms are unclear, the presence of this syndrome is, in some patients, associated with an increased risk of developing subsequent leukemia.

\subsection{Future developments}

Recent developments in the use of glycan arrays for systematic screening of blood samples has lead to the discovery of a panel of anti-glycan antibodies which may prove useful as biomarkers enabling better diagnosis and prognosis of diseases such as Crohn's disease and multiple sclerosis (MS).

\subsubsection{Anti-Glycan Abs as biomarkers for $C D$}

One of the major serological markers for $\mathrm{CD}$ is anti-Sacharomyces cerevisiae Abs, which is directed against oligomannosidic residues on the polysaccharide mannan in the cell walls of the yeast $S$. cerevisi$a e$. Recent systematic screening for anti-glycan antibodies in CD using glycan array have lead to the discovery of novel anti-glycan abs [83]. These include anti-laminaribioside $(\operatorname{Glc}(\beta 1,3) \operatorname{Glc}(\beta))$ and antimannobioside $(\operatorname{Man}(\alpha 1,3) \operatorname{Man}(\alpha))$ glycan IgG Abs, as well as anti-chitobioside $(\operatorname{GlcNAc}(\beta 1,4) \operatorname{GlcNAc}(\beta))$ glycan $\operatorname{IgA}$ Abs; with the latter demonstrating the highest discriminative capability between CD and UC. Combination of these anti-glycan biomarkers have been shown to be useful prognostic tool; predicting severe and complicated CD (presence of strictures or fistulas) and the need for surgical intervention.

\subsubsection{Anti-Glycan Abs as biomarkers for diagnosis of $M S$}

MS is an inflammatory demyelinating disease of the central nervous system. The disease is autoimmune in nature and is driven by a primary T-celldriven aberrant immune response, as well as an antigen driven B-cell responses. The panel of antibodies include a notable number of anti glycan antibodies, anti-galactocerebroside $\operatorname{IgG}$ and anti-Glc $(\alpha 1,4) \operatorname{Glc}(\alpha)$ $\operatorname{IgM}$ auto-Abs. The levels of the latter were found to be significantly elevated in MS patients in comparison to other neurological diseases (with a $57 \%$ sensitivity and $85 \%$ specificity) [83]. These anti-glyco auto-Abs maybe particularly useful for the early diagnosis and prognosis of the relapsing - remitting form of MS.

\section{Conclusion}

There is a pressing need to develop new biomarkers that will serve as more sensitive diagnostic and prognostic tools which could be used to discriminate between different forms/stages of disease and to monitor the efficacy of various new treatment options. Glycobiomarkers have the potential to fulfill this need by providing better link between specific mRNAs, their corresponding polypeptides, glycoforms and cell function, and may thus provide a better insight into cellular and molecular interactions and therefore disease mechanisms; promising a new era in the interpretation of data relevant to immunotherapy and the design of new oligosaccharide-based diagnostics and therapeutics.

\section{Abbreviations}

ABO antigens: $\mathrm{ABO}$ histo-blood group antigens

AutoAbs: Auto antibodies

bis-GlcNAc: bisecting $N$-acetylglucosamine

CD: Crohn's disease

CDGs: congenital disorders of glycosylation

G0: agalactosyl

G1: monogalactosyl

$\mathrm{G} 2$ : digalactosyl

G0F: fucosylated G0

HSP: Henoch-Schönlein Purpura

IBD: Inflammatory Bowel Disease

IgA: immunoglobulin A

IgAN: $\operatorname{Ig}$ A nephropathy

IgG: immunoglobulin $\mathrm{G}$

JIA: juvenile idiopathic arthritis 
MBL: mannose binding lectin

$O$-GlcNAc: $O$-linked $\beta-N$-acetylglucosamine

RA: rheumatoid arthritis

RF: rheumatoid factor

SLE: systemic lupus erythematosus

SS: primary Sjogren's syndrome

UC: ulcerative colitis

\section{References}

[1] P.M. Rudd, T. Elliott, P. Cresswell, I.A. Wilson and R.A Dwek, Glycosylation and the immune system, Science 291(5512) (2001), 2370-2376.

[2] J. Axford, The impact of glycobiology on medicine, Trends Immunol 22(5) (2001), 237-239.

[3] A. Alavi and J.S. Axford, The pivotal nature of sugars in normal physiology and disease, Wien Med Wochenschr 156(1-2) (2006), 19-33.

[4] J.S.E. Axford, ed., Glycobiology and Medicine, Proceedings of the 7th Jenner Glycobiology and Medicine Symposium, Springer, 2005.

[5] B.A. Cobb and D.L. Kasper, Coming of age: carbohydrates and immunity, Eur J Immunol 35(2) (2005), 352-356.

[6] N.H. Packer, C.W. von der Lieth, K.F. Aoki-Kinoshita, C.B. Lebrilla, J.C. Paulson, R. Raman et al., Frontiers in glycomics: bioinformatics and biomarkers in disease. An NIH White Paper prepared from discussions by the focus groups at a workshop on the NIH campus, Bethesda MD (September 11-13, 2006), Proteomics 8(1) (2008), 8-20.

[7] A. Varki, Nothing in glycobiology makes sense, except in the light of evolution, Cell 126(5) (2006), 841-845.

[8] H.J. Gabius, Cell surface glycans: the why and how of their functionality as biochemical signals in lectin-mediated information transfer, Crit Rev Immunol 26(1) (2006), 43-79.

[9] E.I. Buzas, B. Gyorgy, M. Pasztoi, I. Jelinek, A. Falus and H.J. Gabius, Carbohydrate recognition systems in autoimmunity, Autoimmunity 39(8) (2006), 691-704.

[10] J.D. Hernandez, J.T. Nguyen, J. He, W. Wang, B. Ardman, J.M. Green et al., Galectin-1 binds different CD43 glycoforms to cluster CD43 and regulate T cell death, J Immunol 177(8) (2006), 5328-5336.

[11] S. Nakahara and A. Raz, Biological modulation by lectins and their ligands in tumor progression and metastasis, Anticancer Agents Med Chem 8(1) (2008), 22-36.

[12] P.R. Crocker, J.C. Paulson and A. Varki, Siglecs and their Roles in the Immune System 7(4) (2007), 255-266.

[13] E.M. Egorina, M.A. Sovershaev and B. Osterud, Regulation of tissue factor procoagulant activity by post-translational modifications, Thromb Res, 2008

[14] V. Ilic, N. Milosevic-Jovcic, S. Petrovic, D. Markovic, G. Stefanovic and T. Ristic, Glycosylation of IgG B cell receptor (IgG BCR) in multiple myeloma: relationship between sialylation and the signal activity of IgG BCR, Glycoconj J, 2008.

[15] J. Meng, P. Parroche, D.T. Golenbock and C.J. McKnight, The differential impact of disulfide bonds and N-linked glycosylation on the stability and function of CD14, J Biol Chem 283(6) (2008), 3376-3384.

[16] X. Yang, J. Yip, M. Harrison and I. Brockhausen, Primary human osteoblasts and bone cancer cells as models to study glycodynamics in bone, Int J Biochem Cell Biol 40(3) (2008), 471-483.

[17] A. Golks, T.T. Tran, J.F. Goetschy and D. Guerini, Requirement for O-linked N-acetylglucosaminyltransferase in lymphocytes activation, Embo J 26(20) (2007), 4368-4379.

[18] M.A. Toscano, G.A. Bianco, J.M. Ilarregui, D.O. Croci, J. Correale, J.D. Hernandez et al., Differential glycosylation of TH1, TH2 and TH-17 effector cells selectively regulates susceptibility to cell death, Nat Immunol 8(8) (2007), 825834.

[19] J. Jenner, G. Kerst, R. Handgretinger and I. Muller, Increased alpha2,6-sialylation of surface proteins on tolerogenic, immature dendritic cells and regulatory T cells, Exp Hematol 34(9) (2006), 1212-1218.

[20] L. Medvedova and R. Farkas, Hormonal control of protein glycosylation: role of steroids and related lipophilic ligands, Endocr Regul 38(2) (2004), 65-79.

[21] A. Alavi and J. Axford, The Glycosyltransferases, in: Abnormalities of IgG Glycosylation and Immunological Disorders, D. Isenberg, Radmaecher, eds, 1996, pp. 149-169.

[22] K.S. Lau, E.A. Partridge, A. Grigorian, C.I. Silvescu, V.N. Reinhold, M. Demetriou et al., Complex N-glycan number and degree of branching cooperate to regulate cell proliferation and differentiation, Cell 129(1) (2007), 123-134.

[23] V.S. Sarafian and T.T. Marinova, ABH histo-blood group antigens in human thymus involution, Arch Med Res 37(7) (2006), 844-847.

[24] K.S. Nandakumar, M. Collin, A. Olsen, F. Nimmerjahn, A.M. Blom, J.V. Ravetch et al., Endoglycosidase treatment abrogates IgG arthritogenicity: importance of $\mathrm{IgG}$ glycosylation in arthritis, Eur J Immunol 37(10) (2007), 2973-2982.

[25] J.J. Caramelo and A.J. Parodi, How sugars convey information on protein conformation in the endoplasmic reticulum, Semin Cell Dev Biol 18(6) (2007), 732-742.

[26] M. Aridor, Visiting the ER: the endoplasmic reticulum as a target for therapeutics in traffic related diseases, Adv Drug Deliv Rev 59(8) (2007), 759-781.

[27] O. Vagin, S. Turdikulova and E. Tokhtaeva, Polarized membrane distribution of potassium-dependent ion pumps in epithelial cells: different roles of the $\mathrm{N}$-glycans of their beta subunits, Cell Biochem Biophys 47(3) (2007), 376-391.

[28] R. Stork, K.A. Zettlitz, D. Muller, M. Rether, F.G. Hanisch and R.E. Kontermann, N-glycosylation as novel strategy to improve pharmacokinetic properties of bispecific singlechain diabodies, J Biol Chem, 2008.

[29] Y. Li, B. Cleveland, I. Klots, B. Travis, B.A. Richardson, D. Anderson et al., Removal of a single N-linked glycan in human immunodeficiency virus type 1 gp120 results in an enhanced ability to induce neutralizing antibody responses, J Virol 82(2) (2008), 638-651.

[30] G. Opdenakker, C. Dillen, P. Fiten, E. Martens, I. van Aelst, P.E. van den Steen et al., Remnant epitopes, autoimmunity and glycosylation, Biochim Biophys Acta 1760(4) (2006), 610-615.

[31] G.A. Rabinovich, M.A. Toscano, S.S. Jackson and G.R. Vasta, Functions of cell surface galectin-glycoprotein lattices, Curr Opin Struct Biol 17(5) (2007), 513-520.

[32] M.V. Tribulatti, J. Mucci, V. Cattaneo, F. Aguero, T. Gilmartin, S.R. Head et al., Galectin-8 induces apoptosis in the CD4(high)CD8(high) thymocyte subpopulation, Glycobiology 17(12) (2007), 1404-1412.

[33] F. Nimmerjahn and J.V. Ravetch, Fc-receptors as regulators of immunity, Adv Immunol 96 (2007), 179-204. 
[34] M. Bax, J.J. Garcia-Vallejo, J. Jang-Lee, S.J. North, T.J. Gilmartin, G. Hernandez et al., Dendritic cell maturation results in pronounced changes in glycan expression affecting recognition by siglecs and galectins, J Immunol 179(12) (2007), 8216-8224.

[35] E. Balcan, I. Tuglu, M. Sahin and P. Toparlak, Cell surface glycosylation diversity of embryonic thymic tissues, Acta Histochem 110(1) (2008), 14-25.

[36] O. Vagin, E. Tokhtaeva, I. Yakubov, E. Shevchenko and G. Sachs, Inverse correlation between the extent of N-glycan branching and intercellular adhesion in epithelia, Contribution of the Na,K-ATPase beta1 subunit, J Biol Chem 283(4) (2008), 2192-2202.

[37] J.L. Miller, B.J. Dewet, L. Martinez-Pomares, C.M. Radcliffe, R.A. Dwek, P.M. Rudd et al., The Mannose Receptor Mediates Dengue Virus Infection of Macrophages, PLoS Pathog 4(2) (2008), e17.

[38] C. Sugimoto, E.E. Nakayama, T. Shioda, F. Villinger, A.A. Ansari, N. Yamamoto et al., Impact of glycosylation on antigenicity of simian immunodeficiency virus SIV239: induction of rapid V1/V2-specific non-neutralizing antibody and delayed neutralizing antibody following infection with an attenuated deglycosylated mutant, J Gen Virol 89(Pt 2) (2008), $554-566$.

[39] S. Fauquenoy, W. Morelle, A. Hovasse, A. Bednarczyk, C. Slomianny, C. Schaeffer et al., Proteomic and glycomic analyses of N-glycosylated structures involved in toxoplasma gondii-host cell interactions, Mol Cell Proteomics, 2008.

[40] S. Groux-Degroote, M.A. Krzewinski-Recchi, A. Cazet, A. Vincent, S. Lehoux, J.J. Lafitte et al., IL-6 and IL-8 increase the expression of glycosyltransferases and sulfotransferases involved in the biosynthesis of sialylated and/or sulfated Lewisx epitopes in the human bronchial mucosa, Biochem $J$ 410(1) (2008), 213-223.

[41] G. Garratty, Blood groups and disease: a historical perspective, Transfus Med Rev 14(4) (2000), 291-301.

[42] M. Tan and X. Jiang, Norovirus-host interaction: implications for disease control and prevention, Expert Rev Mol Med 9(19) (2007), 1-22.

[43] L.C. Lindesmith, E.F. Donaldson, A.D. Lobue, J.L. Cannon, D.P. Zheng, J. Vinje et al., Mechanisms of GII.4 norovirus persistence in human populations, PLoS Med 5(2) (2008), e31.

[44] S. Linden, J. Mahdavi, C. Semino-Mora, C. Olsen, I. Carlstedt, T. Boren et al., Role of ABO secretor status in mucosal innate immunity and $\mathrm{H}$. pylori infection, PLoS Pathog 4(1) (2008), e2.

[45] M.P. Loscertales, S. Owens, J. O'Donnell, J. Bunn, X. BoschCapblanch and B.J. Brabin, ABO blood group phenotypes and Plasmodium falciparum malaria: unlocking a pivotal mechanism, Adv Parasitol 65 (2007), 1-50.

[46] O. Wu, N. Bayoumi, M.A. Vickers and P. Clark, $\mathrm{ABO}(\mathrm{H})$ blood groups and vascular disease: a systematic review and meta-analysis, J Thromb Haemost 6(1) (2008), 62-69.

[47] E. Dabelsteen and S. Gao, ABO blood-group antigens in oral cancer, J Dent Res 84(1) (2005), 21-28.

[48] S. Marionneau, A. Cailleau-Thomas, J. Rocher, B. Le Moullac-Vaidye, N. Ruvoen, M. Clement et al., ABH and Lewis histo-blood group antigens, a model for the meaning of oligosaccharide diversity in the face of a changing world, Biochimie 83(7) (2001), 565-573.

[49] J.C. Chatham, L.G. Not, N. Fulop and R.B. Marchase, Hexosamine Biosynthesis and Protein O-Glycosylation: The
First Line of Defense against Stress, Ischemia, and Trauma, Shock, 2007.

[50] J.E. Rexach, P.M. Clark and L.C. Hsieh-Wilson, Chemical approaches to understanding O-GlcNAc glycosylation in the brain, Nat Chem Biol 4(2) (2008), 97-106.

[51] R. Morgan, G. Gao, J. Pawling, J.W. Dennis, M. Demetriou and B. Li, N-acetylglucosaminyltransferase V (Mgat5)mediated $\mathrm{N}$-glycosylation negatively regulates $\mathrm{Th} 1$ cytokine production by T cells, J Immunol 173(12) (2004), 72007208.

[52] V. Vanhooren, L. Desmyter, X.E. Liu, M. Cardelli, C. Franceschi, A. Federico et al., N-glycomic changes in serum proteins during human aging, Rejuvenation Res 10(4) (2007), 521-531a.

[53] P. Cheung, J. Pawling, E.A. Partridge, B. Sukhu, M. Grynpas and J.W. Dennis, Metabolic homeostasis and tissue renewal are dependent on beta1,6GlcNAc-branched N-glycans, Glycobiology 17(8) (2007), 828-837.

[54] S.A. Brooks, T.M. Carter, L. Royle, D.J. Harvey, S.A. Fry, C. Kinch et al., Altered glycosylation of proteins in cancer: what is the potential for new anti-tumour strategies, Anticancer Agents Med Chem 8(1) (2008), 2-21.

[55] M. Watson, P.M. Rudd, M. Bland, R.A. Dwek and J.S. Axford, Sugar printing rheumatic diseases: a potential method for disease differentiation using immunoglobulin $\mathrm{G}$ oligosaccharides, Arthritis Rheum 42(8) (1999), 1682-1690.

[56] K. Martin, R. Talukder, F.C. Hay and J.S. Axford, Characterization of changes in IgG associated oligosaccharide profiles in rheumatoid arthritis, psoriatic arthritis, and ankylosing spondylitis using fluorophore linked carbohydrate electrophoresis, J Rheumatol 28(7) (2001), 1531-1536.

[57] J.S. Axford, G. Cunnane, O. Fitzgerald, J.M. Bland, B. Bresnihan and E.R. Frears, Rheumatic disease differentiation using immunoglobulin $\mathrm{G}$ sugar printing by high density electrophoresis, J Rheumatol 30(12) (2003), 2540-2546.

[58] A. Alavi and J. Axford, Sweet \& Sour: The impact of sugars on disease, Rheumatol, In Press, 2008.

[59] L.J. Marshall, B. Perks, K. Bodey, R. Suri, A. Bush and J.K. Shute, Free secretory component from cystic fibrosis sputa displays the cystic fibrosis glycosylation phenotype, Am J Respir Crit Care Med 169(3) (2004), 399-406.

[60] V.A. Lawson, S.J. Collins, C.L. Masters and A.F. Hill, Prion protein glycosylation, J Neurochem 93(4) (2005), 793-801.

[61] T. Lefebvre, C. Guinez, V. Dehennaut, O. Beseme-Dekeyser, W. Morelle and J.C. Michalski, Does O-GlcNAc play a role in neurodegenerative diseases? Expert Rev Proteomics 2(2) (2005), 265-275.

[62] P.T. Martin, Congenital muscular dystrophies involving the O-mannose pathway, Curr Mol Med 7(4) (2007), 417-425.

[63] R.S. Bakri, B. Afzali, A. Covic, R. Sriskantharan, P. BharmaAriza, W.H. Park et al., Cardiovascular disease in renal allograft recipients is associated with elevated sialic acid or markers of inflammation, Clin Transplant 18(2) (2004), 201204.

[64] N. Fulop, R.B. Marchase and J.C. Chatham, Role of protein $\mathrm{O}$-linked $\mathrm{N}$-acetyl-glucosamine in mediating cell function and survival in the cardiovascular system, Cardiovasc Res 73(2) (2007), 288-297.

[65] K. Bodger, J. Halfvarson, A.R. Dodson, F. Campbell, S. Wilson, R. Lee et al., Altered colonic glycoprotein expression in unaffected monozygotic twins of inflammatory bowel disease patients, Gut 55(7) (2006), 973-977. 
[66] J. Novak, B.A. Julian, M. Tomana and J. Mestecky, IgA glycosylation and IgA immune complexes in the pathogenesis of IgA nephropathy, Semin Nephrol 28(1) (2008), 78-87.

[67] A.G. Gharavi, Z. Moldoveanu, R.J. Wyatt, C.V. Barker, S.Y. Woodford, R.P. Lifton et al., Aberrant IgA1 Glycosylation Is Inherited in Familial and Sporadic IgA Nephropathy, $J$ Am Soc Nephrol, 2008.

[68] K.S. Weber, R. Alon and L.B. Klickstein, Sialylation of ICAM-2 on platelets impairs adhesion of leukocytes via LFA1 and DC-SIGN, Inflammation 28(4) (2004), 177-188.

[69] C. Willyard, Researchers look for 'sweet' method to diagnose cancer, Nat Med 13(11) (2007), 1267.

[70] Y. Qiu, T.H. Patwa, L. Xu, K. Shedden, D.E. Misek, M. Tuck et al., Plasma Glycoprotein Profiling for Colorectal Cancer Biomarker Identification by Lectin Glycoarray and Lectin Blot, J Proteome Res, 2008.

[71] S.J. Storr, L. Royle, C.J. Chapman, U.M. Hamid, J.F. Robertson, A. Murray et al., The O-linked glycosylation of secretory/shed MUC1 from advanced breast cancer patient serum, Glycobiology, 2008.

[72] J. Jaeken and G. Matthijs, Congenital disorders of glycosylation: a rapidly expanding disease family, Аппи Rev Genomics Hum Genet 8 (2007), 261-278.

[73] T. Okanishi, Y. Saito, I. Yuasa, M. Miura, I. Nagata, Y. Maegaki et al., Cutis laxa with frontoparietal cortical malformation: A novel type of congenital disorder of glycosylation, Eur J Paediatr Neurol, 2008.

[74] C. Perez-Cerda, D. Quelhas, A.I. Vega, J. Ecay, L. Vilarinho and M. Ugarte, Screening using serum percentage of carbohydrate-deficient transferrin for congenital disorders of glycosylation in children with suspected metabolic disease, Clin Chem 54(1) (2008), 93-100.

[75] J. Gu and M. Taniguchi, Regulation of integrin functions by N-glycans, Glycoconj J 21(1-2) (2004), 9-15.

[76] L.B. Lowe, Glycosylation in the control of selectin counterreceptor structure and function, Immunol Rev $\mathbf{8 6}$ (2002), 1936.

[77] M. Kilcoyne and L. Joshi, Carbohydrates in therapeutics, Cardiovasc Hematol Agents Med Chem 5(3) (2007), 186197.

[78] M. Tatsumoto, M. Koga, M. Gilbert, M. Odaka, K. Hirata, S. Kuwabara et al., Spectrum of neurological diseases associated with antibodies to minor gangliosides GM1b and GalNAc-GD1a, J Neuroimmunol 177(1-2) (2006), 201-208.

[79] D. Chui, G. Sellakumar, R. Green, M. Sutton-Smith, T. McQuistan, K. Marek et al., Genetic remodeling of protein glycosylation in vivo induces autoimmune disease, Proc Natl Acad Sci USA 98(3) (2001), 1142-1147.

[80] H.A. Doyle and M.J. Mamula, Post-translational protein modifications in antigen recognition and autoimmunity, Trends Immunol 22(8) (2001), 443-439.

[81] F. Altmann, The role of protein glycosylation in allergy, Int Arch Allergy Immunol 142(2) (2007), 99-115.

[82] D. Wu, M. Fujio and C.H. Wong, Glycolipids as immunostimulating agents, Bioorg Med Chem 16(3) (2008), 10731083.

[83] I. Dotan, S. Fishman, Y. Dgani, M. Schwartz, A. Karban, A. Lerner et al., Antibodies against laminaribioside and chitobioside are novel serologic markers in Crohn's disease, Gastroenterology 131(2) (2006), 366-378.

[84] D.H. Dube and C.R. Bertozzi, Glycans in cancer and inflammation-potential for therapeutics and diagnostics, Nat Rev Drug Discov 4(6) (2005), 477-488.
[85] M. Bardor, D.H. Nguyen, S. Diaz and A. Varki, Mechanism of uptake and incorporation of the non-human sialic acid $\mathrm{N}$-glycolylneuraminic acid into human cells, J Biol Chem 280(6) (2005), 4228-4237.

[86] R. Parekh, D. Isenberg, G. Rook, I. Roitt, R. Dwek and T. Rademacher, A comparative analysis of disease-associated changes in the galactosylation of serum $\mathrm{IgG}, J$ Autoimmun 2(2) (1989), 101-114.

[87] Y. Kaneko, F. Nimmerjahn and J.V. Ravetch, Antiinflammatory activity of immunoglobulin $\mathrm{G}$ resulting from Fc sialylation, Science 313(5787) (2006), 670-673.

[88] R. Jefferis, Antibody therapeutics: isotype and glycoform selection, Expert Opin Biol Ther 7(9) (2007), 1401-1413.

[89] J.S. Axford, L. Mackenzie, P.M. Lydyard, F.C. Hay, D.A. Isenberg and I.M. Roitt, Reduced B-cell galactosyltransferase activity in rheumatoid arthritis, Lancet 2(8574) (1987), 1486-1488.

[90] J.S. Axford, N. Sumar, A. Alavi, D.A. Isenberg, A. Young, K.B. Bodman et al., Changes in normal glycosylation mechanisms in autoimmune rheumatic disease, J Clin Invest 89(3) (1992), 1021-1031.

[91] A. Alavi, N. Arden, T.D. Spector and J.S. Axford, Immunoglobulin $G$ glycosylation and clinical outcome in rheumatoid arthritis during pregnancy, J Rheumatol 27(6) (2000), 1379-1385.

[92] A. Matsumoto, K. Shikata, F. Takeuchi, N. Kojima and T. Mizuochi, Autoantibody activity of IgG rheumatoid factor increases with decreasing levels of galactosylation and sialylation, J Biochem (Tokyo) 128(4) (2000), 621-628.

[93] C.T. Chou, Binding of rheumatoid and lupus synovial fluids and sera-derived human IgG rheumatoid factor to degalactosylated IgG, Arch Med Res 33(6) (2002), 541-544.

[94] A. Alavi, J.S. Axford and A.J. Pool, Serum galactosyltransferase isoform changes in rheumatoid arthritis, J Rheumatol 31(8) (2004), 1513-1520.

[95] K. Masuda, Y. Yamaguchi, K. Kato, N. Takahashi, I. Shimada and Y. Arata, Pairing of oligosaccharides in the Fc region of immunoglobulin G, FEBS Lett 473(3) (2000), 349-357.

[96] Y. Kuroda, M. Nakata, A. Makino, A. Matsumoto, K. Ohashi, K. Itahashi et al., Structural studies on IgG oligosaccharides of patients with primary Sjogren's syndrome, Glycoconj $J$ 19(1) (2002), 23-31.

[97] H. Das, T. Atsumi, Y. Fukushima, H. Shibuya, K. Ito, Y. Yamada et al., Diagnostic value of antiagalactosyl IgG antibodies in rheumatoid arthritis, Clin Rheumatol 23(3) (2004), 218-222.

[98] D. van Zeben, G.A. Rook, J.M. Hazes, A.H. Zwinderman, Y. Zhang, S. Ghelani et al., Early agalactosylation of IgG is associated with a more progressive disease course in patients with rheumatoid arthritis: results of a follow-up study, $\mathrm{Br} J$ Rheumatol 33(1) (1994), 36-43.

[99] E. Gindzienska-Sieskiewicz, P.A. Klimiuk, D.G. Kisiel, A. Gindzienski and S. Sierakowski, The changes in monosaccharide composition of immunoglobulin $\mathrm{G}$ in the course of rheumatoid arthritis, Clin Rheumatol 2006.

[100] M. Pasek, M. Duk, M. Podbielska, R. Sokolik, J. Szechinski, E. Lisowska et al., Galactosylation of IgG from rheumatoid arthritis (RA) patients - changes during therapy, Glycoconj $J$ 23(7-8) (2006), 463-471.

[101] B.D. Oortwijn, A. Roos, L. Royle, D.J. van GijlswijkJanssen, M.C. Faber-Krol, J.W. Eijgenraam et al., Differential Glycosylation of Polymeric and Monomeric IgA: A Possible Role in Glomerular Inflammation in IgA Nephropathy, J Am Soc Nephrol, 2006. 
[102] B.J. Campbell, L.G. Yu and J.M. Rhodes, Altered glycosylation in inflammatory bowel disease: a possible role in cancer development, Glycoconj J 18(11-12) (2001), 851-858.

[103] N. Maeno, S. Takei, S. Fujikawa, Y. Yamada, H. Imanaka, M. Hokonohara et al., Antiagalactosyl IgG antibodies in juvenile idiopathic arthritis, juvenile onset Sjogren's syndrome, and healthy children, J Rheumatol 31(6) (2004), 1211-1217.

[104] M.H. Ravindranath, S. Muthugounder, T.S. Saravanan, N Presser and D.L. Morton, Human antiganglioside autoantibodies: validation of ELISA, Ann N Y Acad Sci 1050 (2005), 229-242.

[105] K. Kaida, S. Kusunoki, K. Kamakura, K. Motoyoshi and I.
Kanazawa, GalNAc-GD1a in human peripheral nerve: target sites of anti-ganglioside antibody, Neurology 61(4) (2003), 465-470.

[106] M.L. Kuijf, P.C. Godschalk, M. Gilbert, H.P. Endtz, A.P. TioGillen, C.W. Ang et al., Origin of ganglioside complex antibodies in Guillain-Barre syndrome, J Neuroimmunol 188(12) (2007), 69-73.

[107] O.O. Blumenfeld, P. Lalezari, M. Khorshidi, K. Puglia and M. Fukuda, O-linked oligosaccharides of glycophorins A and $\mathrm{B}$ in erythrocytes of two individuals with the Tn polyagglutinability syndrome, Blood 80(9) (1992), 2388-2395. 


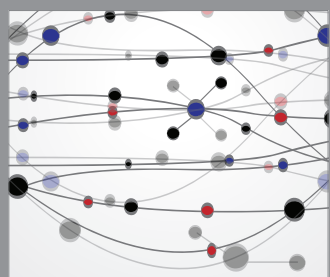

The Scientific World Journal
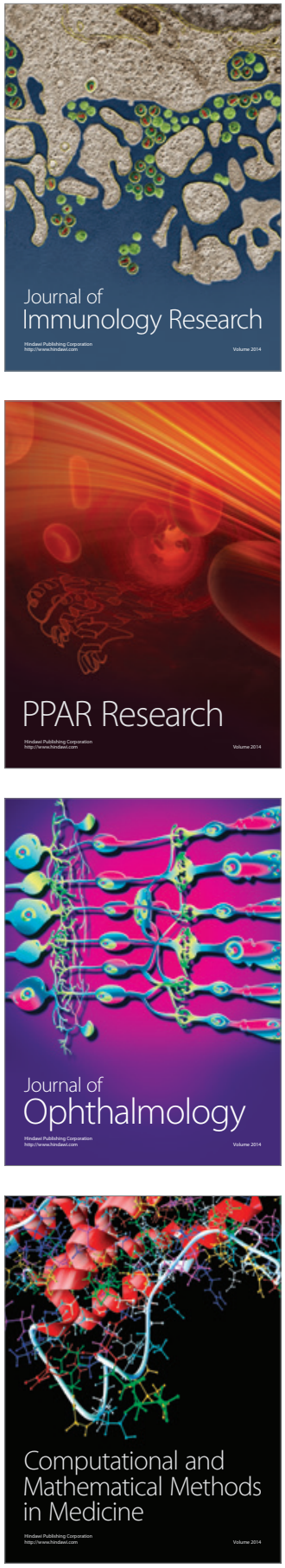

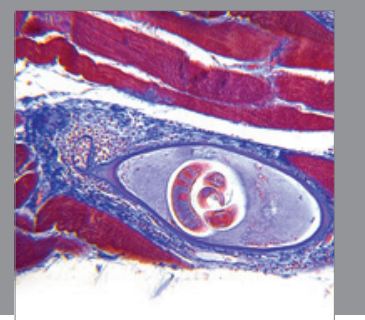

Gastroenterology

Research and Practice
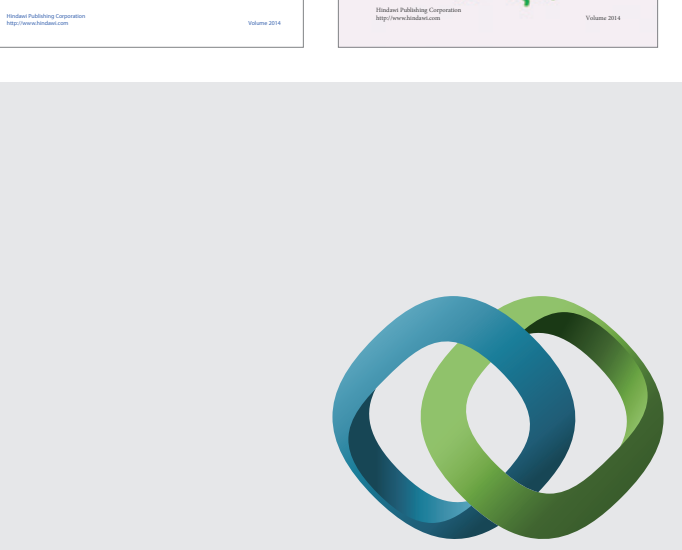

\section{Hindawi}

Submit your manuscripts at

http://www.hindawi.com
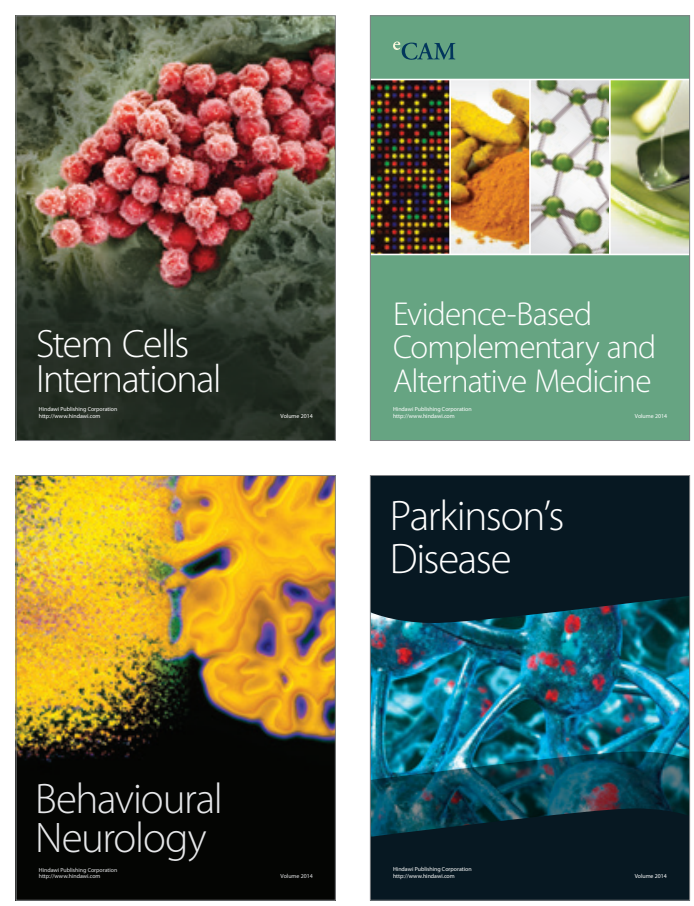

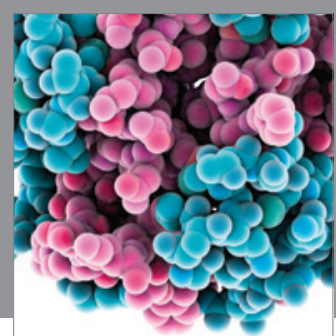

Journal of
Diabetes Research

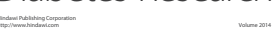

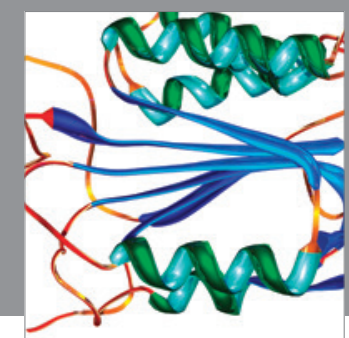

Disease Markers
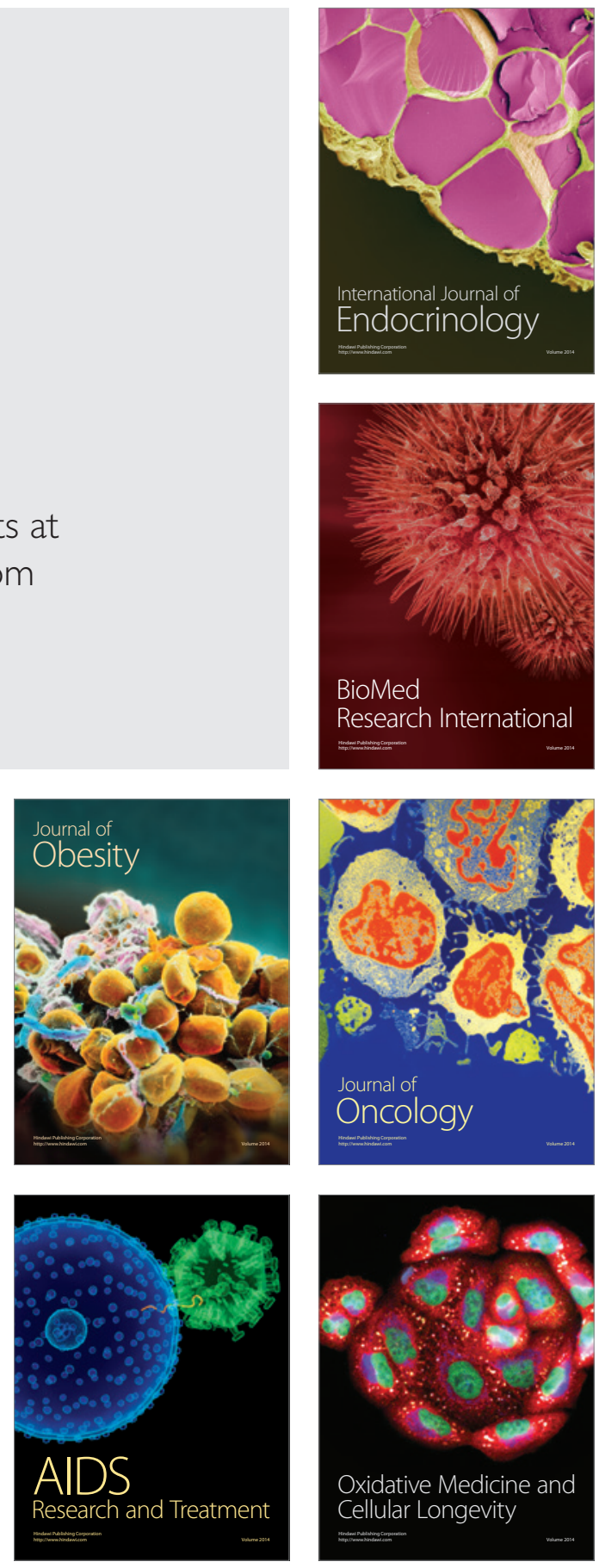